\title{
TESTING FOR THE BUFFERED AUTOREGRESSIVE PROCESSES
}

\author{
Ke Zhu ${ }^{1}$, Philip L. H. Yu ${ }^{2}$ and Wai Keung $\mathrm{Li}^{2}$ \\ ${ }^{1}$ Chinese Academy of Sciences and ${ }^{2}$ University of Hong Kong
}

\begin{abstract}
This paper investigates a quasi-likelihood ratio (LR) test for the thresholds in buffered autoregressive processes. Under the null hypothesis of no threshold, the LR test statistic converges to a function of a centered Gaussian process. Under local alternatives, this LR test has nontrivial asymptotic power. A bootstrap method is proposed to obtain the critical value for the LR test. Simulation studies and an example are given to assess the performance of the test. The proof here is not standard and can be used in other non-linear time series models.
\end{abstract}

Key words and phrases: AR(p) model, bootstrap method, buffered AR(p) model, likelihood ratio test, marked empirical process, threshold $\mathrm{AR}(\mathrm{p})$ model.

\section{Introduction}

After the seminal work of Tlong (1978), threshold autoregressive (TAR) models have achieved great success in practice; see, e.g., Tong (1990) for earlier works and Tong (20II) and the references therein for more recent ones. Generally speaking, the TAR model says that the structure of an AR model shifts among different regimes, i.e.,

$$
y_{t}=\phi_{0}+\sum_{i=1}^{p} \phi_{i} y_{t-i}+\left(\psi_{0}+\sum_{i=1}^{p} \psi_{i} y_{t-i}\right) R_{t}+\varepsilon_{t}
$$

where $R_{t}=I\left(y_{t-d} \leq r\right)$ is the regime indicator of $y_{t}, r$ is the threshold parameter, $d(\geq 1)$ is the delay parameter, and $\varepsilon_{t}$ is an uncorrelated error sequence with zero mean and variance $\sigma^{2}(>0)$. There has been a lot of interest in detecting thresholds in TAR models. Chan (1990, 1991) and Chan and Tong (I.990) first accomplished this by considering a likelihood ratio (LR) test. Tsay (1989) gave some novel methods in this context; Hansen (11996) studied the Wald test and Lagrange multiplier (LM) test for TAR models; Wong and Li (1997, 2000) studied the LM test for TAR-ARCH models; Li and Ling (2013) investigated the portmanteau test for threshold double AR models; see also [say (1998), Hansen (1999), Caner and Hansen (2007), Ling and Tong (2005), Li and Lil (20018, 2017), and Zhu and Ling (2012). 
Under ([.]), the regime of $y_{t}$ shifts when the state of $y_{t-d}$ changes. In practice, the regime of $y_{t}$ may not shift immediately, and there could be a buffering region in which the regime of $y_{t}$ depends on the regime of $y_{t-d}$. Li et al. (2012) first formulated this by assuming that $R_{t}$ in (미) satisfies

$$
R_{t}= \begin{cases}1 & \text { if } y_{t-d} \leq r_{L} \\ 0 & \text { if } y_{t-d}>r_{U} \\ R_{t-1} & \text { otherwise }\end{cases}
$$

where $r_{L}$ and $r_{U}$ are two threshold parameters such that $r_{L} \leq r_{U}$. They called (ㅁ. $)$ - (ए.2) the buffered AR (BAR) model, and the region in which $y_{t-d}$ lies between $r_{L}$ and $r_{U}$ is called the buffering region. Also, they found that the BAR model is the best selected model for the sunspot series in Iong (1990) and the GNP series in Tiao and Tsay (19.94); it may provide us with a new way to understand non-linear time series. However, how to test for BAR models is still unknown, and it is more challenging than testing for TAR models because the regime of $y_{t}$ depends on past observations infinitely far away.

In this paper, we investigate a quasi-LR test for the thresholds in BAR models. Under the null hypothesis of no threshold, the LR test statistic converges to a function of a centered Gaussian process. Under local alternatives, this LR test has nontrivial asymptotic power. Our result contains the one in Chan ([1990) as a special case, but its proof is not standard and different from the proof in that paper. A bootstrap method is proposed to obtain the critical value for the LR test. Simulation studies and an example are given to assess the performance of this LR test.

This paper is organized as follows. Section 2 states our main result on the LR test. Section 3 proposes a bootstrap procedure. The simulation results and an example are given in Section 4. The proofs are provided in the Appendix, which can be found in $\mathrm{Zhu}, \mathrm{Yu}$, and Li (2013). Throughout the paper, $|A|=$ $\left(\operatorname{tr}\left(A^{\prime} A\right)\right)^{1 / 2}$ is the Euclidean norm of a matrix $A,\|A\|_{s}=\left(E|A|^{s}\right)^{1 / s}$ is the $L^{s}$-norm $(s \geq 1)$ of a random matrix, $A^{\prime}$ is the transpose of matrix $A, o_{p}(1)$ $\left(O_{p}(1)\right)$ denotes a sequence of random numbers converging to zero (bounded) in probability, $\rightarrow_{d}$ denotes convergence in distribution, and $\Rightarrow$ denotes weak convergence. $I(\cdot)$ is an indicator function.

\section{Likelihood Ratio Test}

Let $\phi=\left(\phi_{0}, \ldots, \phi_{p}\right)^{\prime}, \psi=\left(\psi_{0}, \ldots, \psi_{p}\right)^{\prime}, \lambda=\left(\phi^{\prime}, \psi^{\prime}\right)^{\prime}, \gamma=\left(r_{L}, r_{U}\right)$, and

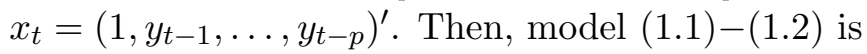

$$
y_{t}=x_{t}(\gamma)^{\prime} \lambda+\varepsilon_{t},
$$


where $x_{t}(\gamma)=\left(x_{t}^{\prime}, h_{t}(\gamma)^{\prime}\right)^{\prime}, h_{t}(\gamma)=x_{t} R_{t}(\gamma)$, and $R_{t}(\gamma)$ is defined as in (प्2). Here, we assume that all the roots of the characteristic equation $\phi(x)=x^{p}-$ $\phi_{1} x^{p-1}-\cdots-\phi_{p}$ lie inside the unit circle, and both $p$ and $d$ are known. We further assume that $d \leq p$ if $p \geq 1$, because we can set $p=d$ with $\phi_{p+1}=\cdots=\phi_{d}=0$ and $\psi_{p+1}=\cdots=\psi_{d}=0$ in (2.]) when $d>p \geq 1$.

Suppose that $\left\{y_{0}, \ldots, y_{N}\right\}$ are $N+1$ consecutive observations from model (ख.]) with the true parameters $\lambda_{0}$ and $\gamma_{0}$, where $\lambda_{0}=\left(\phi_{0}^{\prime}, \psi_{0}^{\prime}\right)^{\prime}, \phi_{0}=\left(\phi_{00}, \ldots, \phi_{p 0}\right)^{\prime}$, $\psi_{0}=\left(\psi_{00}, \ldots, \psi_{p 0}\right)^{\prime}$, and $\gamma_{0}=\left(r_{L 0}, r_{U 0}\right)$. We consider the hypotheses

$$
\left\{\begin{array}{l}
H_{0}: \psi_{0}=0 \\
H_{1}: \psi_{0} \neq 0 \text { for some } \gamma
\end{array}\right.
$$

Model ([.]) is an $\operatorname{AR}(p)$ model under $H_{0}$ and it is a buffered $\operatorname{AR}(p)(\operatorname{BAR}(p))$ model under $H_{1}$. When $r_{L}=r_{U}$ (i.e., the buffering region is absent), (ए2. $)$ is for testing the threshold in the threshold $\operatorname{AR}(p)$ (TAR $(p))$ model, for which the likelihood ratio (LR) test was studied by Chan (ए9.90, ए9.9]) when $\varepsilon_{t} \sim N(0,1)$ is a sequence of i.i.d. random variables. When $r_{L} \neq r_{U}$, since

$$
\begin{aligned}
R_{t}(\gamma)= & I\left(y_{t-d} \leq r_{L}\right) \\
& +\sum_{j=1}^{\infty} I\left(y_{t-j-d} \leq r_{L}\right) \prod_{i=1}^{j} I\left(r_{L}<y_{t-i+1-d} \leq r_{U}\right) \text { a.s. }
\end{aligned}
$$

we see that $R_{t}(\gamma)$ depends on all past observations. The $R_{t}(\gamma)$ in Chan (1990) only depends on $y_{t-d}$, so the test there is not a LR test and may be less powerful. We consider an alternative LR test for (2.2).

Let $Y=\left(y_{p}, \ldots, y_{N}\right)^{\prime}$ and $Z_{\gamma}=\left(X, X_{\gamma}\right)=\left(x_{p}(\gamma), x_{p+1}(\gamma), \ldots, x_{N}(\gamma)\right)^{\prime}$, where $X=\left(x_{p}, x_{p+1}, \ldots, x_{N}\right)^{\prime}$ and $X_{\gamma}=\left(h_{p}(\gamma), h_{p+1}(\gamma), \ldots, h_{N}(\gamma)\right)^{\prime}$. Let $n=$ $N-p+1$ be the effective number of observations. Following Chan (1990), we know that for any fixed value of $\gamma$ the LR test statistic is

where

$$
L R_{n}(\gamma)=\frac{n\left[\sigma_{n}^{2}-\sigma_{n}^{2}(\gamma)\right]}{\sigma_{n}^{2}}
$$

$$
\begin{aligned}
\sigma_{n}^{2} & =\frac{1}{n}\left\{Y^{\prime} Y-\left(Y^{\prime} X\right)\left(X^{\prime} X\right)^{-1}\left(X^{\prime} Y\right)\right\}, \\
\sigma_{n}^{2}(\gamma) & =\frac{1}{n}\left\{Y^{\prime} Y-\left(Y^{\prime} Z_{\gamma}\right)\left(Z_{\gamma}^{\prime} Z_{\gamma}\right)^{-1}\left(Z_{\gamma}^{\prime} Y\right)\right\} .
\end{aligned}
$$

Since the exact value of $\gamma$ is unknown under $H_{0}$, it is natural to construct the LR test by using the maximum of $L R_{n}(\gamma)$ over the range of $\gamma$, see Davies (1.977, 1987). Thus, our LR test statistic is

$$
L R_{n}=\sup _{\gamma \in \Gamma} L R_{n}(\gamma)
$$


where $\Gamma \equiv\left\{\left(r_{L}, r_{U}\right) ; a \leq r_{L} \leq r_{U} \leq b\right\}$ and $[a, b]$ is a predetermined interval. Here, we truncate the full range of $\gamma$, since $L R_{n}$ may diverge to infinity in probability as $n \rightarrow \infty$, see Andrews (1993.3a).

Let $K_{\gamma \delta}=E\left[x_{t}(\gamma) x_{t}(\delta)^{\prime}\right]$. For the asymptotic theory of $L R_{n}$, we need certain technical assumptions.

Assumption 1. $y_{t}$ is strictly stationary, ergodic and absolutely regular with mixing coefficients $\beta(m)=O\left(m^{-A}\right)$ for some $A>v /(v-1)$ and $r>v>1$; $E\left|y_{t}\right|^{4 r}<\infty, E\left|\varepsilon_{t}\right|^{4 r}<\infty$, and $K_{\gamma \gamma}$ is positive definite.

Assumption 2. $y_{t}$ has a bounded and continuous density function.

Assumption 3. There exists an $A_{0}>1$ such that $2 A_{0} r v /(r-v)<A$.

Assumptions 1-2 are from Hansen (1996), where the weak convergence of the empirical process was derived by using the method in Doukhan, Massart, and Rio ([1995). When $\sum_{i=1}^{p}\left|\phi_{i}\right|<1$ and $\sum_{i=1}^{p}\left|\phi_{i}+\psi_{i}\right|<1$, Li et al. (2012) showed that model ([2.]) is strictly stationary and ergodic. When $A>v /(v-1)$, a sufficient condition for Assumption 3 is that $v<3 r /(2 r+1)$, which is stronger than $v<r$ as required in Assumption 1. Particularly, when $\varepsilon_{t}$ is a sequence of i.i.d. random variables with a bounded and continuous density function, $\beta(m)$ decays exponentially under $H_{0}$ as shown in Pham and Tran (198.5). Thus, Assumptions $1-3$ hold in this case.

We state two key lemmas, under which a uniform expansion of $L R_{n}(\gamma)$ can be derived.

Lemma 1. If Assumptions $1-3$ hold, then

(i) $\sup _{\gamma \in \Gamma}\left|\left\{\frac{X_{\gamma}^{\prime} X_{\gamma}}{n}-\frac{X_{\gamma}^{\prime} X}{n}\left(\frac{X^{\prime} X}{n}\right)^{-1} \frac{X^{\prime} X_{\gamma}}{n}\right\}^{-1}-\left(\Sigma_{\gamma}-\Sigma_{\gamma} \Sigma^{-1} \Sigma_{\gamma}\right)^{-1}\right|=o_{p}(1)$;

(ii) under $H_{0}$,

$$
\sup _{\gamma \in \Gamma}\left|T_{\gamma}-\left(-\Sigma_{\gamma} \Sigma^{-1}, I\right) \frac{1}{\sqrt{n}} Z_{\gamma}^{\prime} \varepsilon\right|=o_{p}(1)
$$

where $\varepsilon=\left(\varepsilon_{p}, \ldots, \varepsilon_{N}\right)^{\prime}, T_{\gamma}=n^{-1 / 2}\left\{X_{\gamma}^{\prime}-X_{\gamma}^{\prime} X\left(X^{\prime} X\right)^{-1} X^{\prime}\right\} Y, \Sigma=E\left(x_{t} x_{t}^{\prime}\right)$, and $\Sigma_{\gamma}=E\left[x_{t} x_{t}^{\prime} R_{t}(\gamma)\right]$.

Proof. See the Appendix in Zhu, Yu, and Li (2013).

Lemma 2. If Assumptions 1-3 hold, then

$$
\frac{1}{\sqrt{n}} Z_{\gamma}^{\prime} \varepsilon \Rightarrow \sigma G_{\gamma}
$$

as $n \rightarrow \infty$, where $G_{\gamma}$ is a Gaussian process with zero mean function and covariance $K_{\gamma \delta}$. 
Proof. See the Appendix in Zhu, Yu, and Li (2013).

Note that

$$
\frac{1}{\sqrt{n}} Z_{\gamma}^{\prime} \varepsilon=\frac{1}{\sqrt{n}} \sum_{t=p}^{N}\left(x_{t}^{\prime}, x_{t}^{\prime} R_{t}(\gamma)\right)^{\prime} \varepsilon_{t} .
$$

We call $\left\{n^{-1 / 2} Z_{\gamma}^{\prime} \varepsilon\right\}$ a marked empirical process, as in Stute (1997), where each $y_{t-i-d}$ in $R_{t}(\gamma)$ is a marker. In view of ([2.3),$\left\{n^{-1 / 2} Z_{\gamma}^{\prime} \varepsilon\right\}$ involves infinitely many markers, as Ling and Tong (2015) studied the LR test for TMA models. Their method seems hard to implement here. Compared with Chan (II990) and Ling and Tong (2010.5), the proofs of Lemmas 1-2 in the Appendix are not standard; they may be useful in other non-linear time series models.

Theorem 1. If Assumptions 1-3 hold, then under $H_{0}$,

$$
L R_{n} \rightarrow_{d} \sup _{\gamma \in \Gamma} G_{\gamma}^{\prime} \Omega_{\gamma} G_{\gamma}
$$

as $n \rightarrow \infty$, where $\Omega_{\gamma}=\left(-\Sigma_{\gamma} \Sigma^{-1}, I\right)^{\prime}\left(\Sigma_{\gamma}-\Sigma_{\gamma} \Sigma^{-1} \Sigma_{\gamma}\right)^{-1}\left(-\Sigma_{\gamma} \Sigma^{-1}, I\right)$.

Proof. By $([2.4)-([2.5)$ and a direct calculation,

$$
n\left[\sigma_{n}^{2}-\sigma_{n}^{2}(\gamma)\right]=T_{\gamma}^{\prime}\left\{\frac{X_{\gamma}^{\prime} X_{\gamma}}{n}-\frac{X_{\gamma}^{\prime} X}{n}\left(\frac{X^{\prime} X}{n}\right)^{-1} \frac{X^{\prime} X_{\gamma}}{n}\right\}^{-1} T_{\gamma} .
$$

By Lemmas 1-2, the conclusion follows directly from the argument for Theorem 2.3 in Chan (19901).

Remark 1. Note that

$$
G_{\gamma}^{\prime} \Omega_{\gamma} G_{\gamma}=\xi_{\gamma}^{\prime}\left(\Sigma_{\gamma}-\Sigma_{\gamma} \Sigma^{-1} \Sigma_{\gamma}\right)^{-1} \xi_{\gamma},
$$

where $\xi_{\gamma}=\left(-\Sigma_{\gamma} \Sigma^{-1}, I\right) G_{\gamma}$. Then, by a direct calculation, we can show that, for each $\gamma \in \Gamma, G_{\gamma}^{\prime} \Omega_{\gamma} G_{\gamma}$ follows a $\chi^{2}$ distribution. That is, for fixed $\gamma$, the test statistic $L R_{n}(\gamma)$ is asymptotically pivotal under $H_{0}$.

Remark 2. Although Theorem 1 has Theorem 2.3(ii) of Chan (1.990) as a special case, there has some difference between our LR test and that in Chan (19900). First, the denominator of $L R_{n}(\gamma)$ is different from that in Chan (11990), but the two are asymptotically equivalent; see also Ling and Tong (2010.5). Second, since our $\Gamma$ is larger than that in Chan ([1990), our LR test needs more computational efforts.

Remark 3. As Chan (1990), we only obtained the result under the condition that $\operatorname{Var}\left(\varepsilon_{t}\right)=\sigma^{2}$. The case that the threshold effect is in the variance of $\varepsilon_{t}$ needs further study. 
Next, we study the asymptotical local power of $L R_{n}$ by considering the local alternative hypothesis

$$
H_{1 n}: \psi_{0}=\frac{h}{\sqrt{n}} \text { for a constant vector } h \in \mathcal{R}^{p+1}
$$

Theorem 2. If Assumptions $1-3$ hold, then under $H_{1 n}$,

$$
L R_{n} \rightarrow_{d} \sup _{\gamma \in \Gamma}\left\{G_{\gamma}^{\prime} \Omega_{\gamma} G_{\gamma}+h^{\prime} \mu_{\gamma \gamma_{0}} h\right\}
$$

as $n \rightarrow \infty$, where $M_{\gamma \gamma_{0}}=E\left[x_{t} x_{t}^{\prime} R_{t}(\gamma) R_{t}\left(\gamma_{0}\right)\right]$ and

$$
\mu_{\gamma \gamma_{0}}=\frac{1}{\sigma^{2}}\left(M_{\gamma \gamma_{0}}-\Sigma_{\gamma} \Sigma^{-1} \Sigma_{\gamma}\right)^{\prime}\left(\Sigma_{\gamma}-\Sigma_{\gamma} \Sigma^{-1} \Sigma_{\gamma}\right)^{-1}\left(M_{\gamma \gamma_{0}}-\Sigma_{\gamma} \Sigma^{-1} \Sigma_{\gamma}\right) .
$$

Proof. Note that $Y=X \phi_{0}+X_{\gamma_{0}} h / \sqrt{n}+\varepsilon$ under $H_{1 n}$. Thus,

$$
\begin{aligned}
T_{\gamma} & =\frac{1}{\sqrt{n}}\left\{X_{\gamma}^{\prime}-X_{\gamma}^{\prime} X\left(X^{\prime} X\right)^{-1} X^{\prime}\right\} \varepsilon+\frac{1}{n}\left\{X_{\gamma}^{\prime}-X_{\gamma}^{\prime} X\left(X^{\prime} X\right)^{-1} X^{\prime}\right\} X_{\gamma_{0}} h \\
& =\frac{1}{\sqrt{n}}\left(-\left(X_{\gamma}^{\prime} X\right)\left(X^{\prime} X\right)^{-1}, I\right) Z_{\gamma}^{\prime} \varepsilon+\frac{1}{n}\left\{X_{\gamma}^{\prime}-X_{\gamma}^{\prime} X\left(X^{\prime} X\right)^{-1} X^{\prime}\right\} X_{\gamma_{0}} h .
\end{aligned}
$$

By ([2.6]) and Lemmas 1-2, the conclusion follows directly from the argument for Theorem 2.3 in Chan (1990).

In practice, the values of $a$ and $b$ can be set to empirical quantiles of $\left\{y_{t}\right\}_{t=0}^{N}$ as in Chan (1.991) and Andrews (19.93b), although how to choose the optimal $a, b$ remains unclear. In this case, we can always find a smallest $n_{0} \geq p$ such that $y_{n_{0}-d}$ stays outside the region $[a, b]$, where the integer $n_{0}$ depends on data sample $\left\{y_{0}, \ldots, y_{N}\right\}$. This means that we can observe $R_{n_{0}}(\gamma)$, and then further calculate $\left\{R_{t}(\gamma)\right\}_{t=n_{0}+1}^{N}$ iteratively as

$$
R_{t}(\gamma)=I\left(y_{t-d} \leq r_{L}\right)+R_{t-1} I\left(r_{L}<y_{t-d} \leq r_{U}\right) .
$$

For the remaining observations $\left\{y_{t}\right\}_{t=0}^{n_{0}-1}$ whose regions are not well identified, we set their regions to be 0 . Thus, we can only use $\tilde{R}_{t}(\gamma)$ rather than $R_{t}(\gamma)$ in practice, where

$$
\tilde{R}_{t}(\gamma)=\left\{\begin{array}{cl}
0 & \text { for } t=0, \ldots, n_{0}-1 \\
R_{t}(\gamma) & \text { for } t=n_{0}, \ldots, N
\end{array}\right.
$$

Let $\tilde{L} R_{n}$ be defined in the same way as $L R_{n}$ with $R_{t}(\gamma)$ being replaced by $\tilde{R}_{t}(\gamma)$. The following corollary shows that $\tilde{L R_{n}}$ and $L R_{n}$ have the same asymptotic property. 
Corollary 1. If Assumptions $1-3$ hold, then (i) under $H_{0}$,

$$
\tilde{L R_{n}} \rightarrow_{d} \sup _{\gamma \in \Gamma} G_{\gamma}^{\prime} \Omega_{\gamma} G_{\gamma} \quad \text { as } \quad n \rightarrow \infty
$$

(ii) under $H_{1 n}$,

$$
\tilde{L R_{n}} \rightarrow_{d} \sup _{\gamma \in \Gamma}\left\{G_{\gamma}^{\prime} \Omega_{\gamma} G_{\gamma}+h^{\prime} \mu_{\gamma \gamma_{0}} h\right\} \quad \text { as } n \rightarrow \infty .
$$

Proof. See the Appendix in $\mathrm{Zhu}, \mathrm{Yu}$, and Li (2013).

\section{Bootstrapped Critical Value}

In this section, we use a bootstrap method to obtain the critical value for our LR test; see also Hansen (1996) and $\mathrm{Li}$ and $\mathrm{Lil}(20108$, 201017). First, we let

$$
\hat{\varepsilon}_{t}=y_{t}-x_{t}(\gamma)^{\prime} \lambda_{n}(\gamma)
$$

with

$$
\lambda_{n}(\gamma) \equiv \arg \min _{\lambda \in \Lambda} \sum_{t=p}^{N} \varepsilon_{t}^{2}(\lambda, \gamma)=\left[Z_{\gamma}^{\prime} Z_{\gamma}\right]^{-1}\left[Z_{\gamma}^{\prime} Y\right]
$$

where $\Lambda$ is a compact parametric space of $\lambda$, and $\varepsilon_{t}(\lambda, \gamma)=y_{t}-x_{t}(\gamma)^{\prime} \lambda$. Next, we set

$$
\hat{L R_{n}}(\gamma)=\frac{\hat{Z}_{n}^{\prime}(\gamma)\left(X_{1 n}(\gamma), I\right)^{\prime}\left[X_{2 n}(\gamma)\right]^{-1}\left(X_{1 n}(\gamma), I\right) \hat{Z}_{n}(\gamma)}{\sigma_{n}^{2}}
$$

where $\hat{\varepsilon}=\left(\hat{\varepsilon}_{p} v_{p}, \ldots, \hat{\varepsilon}_{N} v_{N}\right)^{\prime},\left\{v_{t}\right\}_{t=p}^{N}$ is a sequence of i.i.d. $N(0,1)$ random variables, and

$$
\begin{aligned}
\hat{Z}_{n}(\gamma) & =\frac{1}{\sqrt{n}} Z_{\gamma}^{\prime} \hat{\varepsilon}, \quad X_{1 n}(\gamma)=-\frac{X_{\gamma}^{\prime} X}{n}\left(\frac{X^{\prime} X}{n}\right)^{-1}, \\
X_{2 n}(\gamma) & =\frac{X_{\gamma}^{\prime} X_{\gamma}}{n}-\frac{X_{\gamma}^{\prime} X}{n}\left(\frac{X^{\prime} X}{n}\right)^{-1} \frac{X^{\prime} X_{\gamma}}{n} .
\end{aligned}
$$

Define

$$
\hat{L R_{n}} \equiv \sup _{\gamma \in \Gamma} \hat{L R} R_{n}(\gamma)
$$

The asymptotic theory of $\hat{L R_{n}}$ is stated in the following theorem:

Theorem 3. If Assumptions $1-3$ hold, then under $H_{0}$ or $H_{1 n}$,

$$
\hat{L R_{n}} \mid y_{0}, \ldots, y_{N} \rightarrow_{d} \sup _{\gamma \in \Gamma} G_{\gamma}^{\prime} \Omega_{\gamma} G_{\gamma} \quad \text { in probablity as } n \rightarrow \infty \text {. }
$$


Proof. See the Appendix in $\mathrm{Zhu}, \mathrm{Yu}$, and Li (2013).

Remark 4. In practice, $\hat{L R_{n}}$ is calculated with $R_{t}(\gamma)$ being replaced by $\tilde{R}_{t}(\gamma)$. However, by using the argument for Corollary 1, we can show that it does not affect the asymptotic property of $\hat{L R}$.

Note that the conditional limiting distribution in Theorem 3 is the same as the null distribution in Theorem 1 . Then, conditional on the data sample $\left\{y_{0}, \ldots, y_{N}\right\}$ and for given significance level $\alpha$, we use a bootstrap procedure to obtain our critical value:

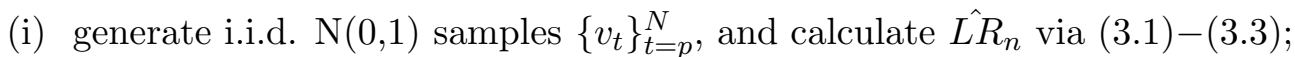

(ii) repeat step (i) $J$ times to get $\left\{\hat{L R_{n}^{(1)}}, \ldots, \hat{L R_{n}^{(J)}}\right\}$;

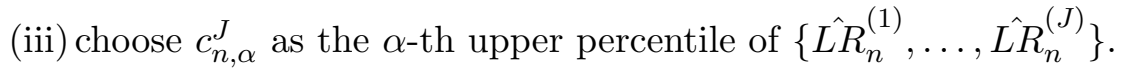

We choose $c_{n, \alpha}^{J}$ as the critical value for our LR test and shorten $c_{n, \alpha}^{J}$ to $c_{n}$ for brevity. In the end, we give a critical corollary as follows:

Corollary 2. If Assumptions $1-3$ hold, then (i) under $H_{0}$,

$$
\lim _{n \rightarrow \infty} \lim _{J \rightarrow \infty} P\left(L R_{n} \geq c_{n, \alpha}^{J}\right)=\alpha
$$

(ii) under $H_{1 n}$,

$$
\lim _{h \rightarrow \infty} \lim _{n \rightarrow \infty} \lim _{J \rightarrow \infty} P\left(L R_{n} \geq c_{n, \alpha}^{J}\right)=1
$$

Proof. See the Appendix in Zhu, Yu, and Li (2013).

Corollary 3.1 guarantees that our bootstrapped critical value $c_{n, \alpha}^{J}$ is asymptotically valid, and our LR test has power to detect $H_{1 n}$. The method can also produce the critical value for the LR test in Chan (1990) by setting $\gamma_{L} \equiv \gamma_{U}$. Since $\hat{L R} R_{n}(\gamma)$ is a step-function, the amount of computation on $c_{n, \alpha}^{J}$ depends only on the effective sample size $n$ and the bootstrapped sample size $J$. This reduces the computational burden significantly.

\section{Simulation and One Real Example}

In this section, we first compare the performance of our LR test $\left(L R_{n}\right)$ and Chan's (1990) LR test $\left(L R_{n}^{*}\right)$ in the finite sample. We generate 1,000 replications of sample size $n=200$ from the BAR model

$$
y_{t}=y_{t-1}-0.09 y_{t-2}+\left(\psi_{1} y_{t-1}+\psi_{2} y_{t-2}\right) R_{t}(\gamma)+\varepsilon_{t},
$$

where $R_{t}(\gamma)$ is defined as in (1.2) with $d=1$, $\varepsilon_{t}$ is $N(0,1)$, and $y_{0}=y_{1}=R_{1}(\gamma)=$ 0 . We choose $\gamma=(0,0),(0,0.5),(0,1.5)$ or $(0,2)$, and use the significance level 
Table 1. Rejection rates.

\begin{tabular}{|c|c|c|c|c|c|c|}
\hline \multicolumn{2}{|c|}{$\psi$} & \multicolumn{2}{|c|}{$\gamma$} & & \multicolumn{2}{|c|}{$L R_{n}^{*}$} \\
\hline$\psi_{1}$ & $\psi_{2}$ & $r_{L}$ & $r_{U}$ & $L R_{n}$ & $L R_{1 n}^{*}$ & $L R_{2 n}^{*}$ \\
\hline 0.0 & 0.0 & - & - & 4.9 & 4.9 & 3.4 \\
\hline \multirow[t]{4}{*}{0.1} & -0.09 & 0.0 & 0.0 & 7.7 & 7.7 & 3.8 \\
\hline & & 0.0 & 0.5 & 7.5 & 7.4 & 3.7 \\
\hline & & 0.0 & 1.5 & 7.6 & 6.5 & 3.2 \\
\hline & & 0.0 & 2.0 & 7.5 & 7.0 & 5.4 \\
\hline \multirow[t]{4}{*}{0.3} & -0.27 & 0.0 & 0.0 & 31.9 & 34.2 & 14.3 \\
\hline & & 0.0 & 0.5 & 30.6 & 30.3 & 16.5 \\
\hline & & 0.0 & 1.5 & 33.4 & 29.6 & 15.4 \\
\hline & & 0.0 & 2.0 & 32.0 & 27.1 & 15.6 \\
\hline \multirow[t]{4}{*}{0.5} & -0.45 & 0.0 & 0.0 & 64.7 & 69.1 & 54.0 \\
\hline & & 0.0 & 0.5 & 76.0 & 79.6 & 55.2 \\
\hline & & 0.0 & 1.5 & 76.1 & 75.5 & 56.0 \\
\hline & & 0.0 & 2.0 & 75.2 & 72.6 & 53.9 \\
\hline \multirow[t]{4}{*}{0.7} & -0.63 & 0.0 & 0.0 & 95.8 & 97.1 & 86.4 \\
\hline & & 0.0 & 0.5 & 89.4 & 90.1 & 89.5 \\
\hline & & 0.0 & 1.5 & 96.0 & 96.0 & 87.8 \\
\hline & & 0.0 & 2.0 & 95.9 & 95.9 & 89.9 \\
\hline
\end{tabular}

$\alpha=0.05$. Since the pair of characteristic roots is $(0.1,0.9)$ in the regime of $R_{t}(\gamma)=0$, we choose $\left(\psi_{1}, \psi_{2}\right)=(0,0),(0.1,-0.09),(0.3,-0.27),(0.5,-0.45)$ or $(0.7,-0.63)$ such that the pair of characteristic roots in the regime of $R_{t}(\gamma)=1$ is $(0.1,0,9),(0.2,0.9),(0.4,0.9),(0.6,0.9)$ or $(0.8,0.9)$, respectively. For each replication, the value of $a$ and $b$ for the interval $[a, b]$ are set as the empirical 10th and 90th quantiles of the data sample, the critical value for $L R_{n}$ is calculated by the bootstrap method in Section 3 with $J=1,000$, and the critical value for $L R_{n}^{*}$ is either calculated in the same way as the one for $L R_{n}$ or taken as 15.18 according to Table 2 in Chan (11991).

Table 1 lists the rejection rates of $L R_{n}$ and $L R_{n}^{*}$ with different values of $\psi$ and $\gamma$. The results for $L R_{n}^{*}$ based on the bootstrapped critical value and Chan's (11991) critical value are denoted by $L R_{1 n}^{*}$ and $L R_{2 n}^{*}$, respectively. The sizes of these tests correspond to the case $\left(\psi_{1}, \psi_{2}\right)=(0,0)$. From Table 1, we find that the sizes of $L R_{n}$ and $L R_{1 n}^{*}$ are close to their nominal levels, but the size of $L R_{2 n}^{*}$ is conservative. Although the power of all tests becomes larger as the two regimes for $R_{t}(\gamma)=0$ and $R_{t}(\gamma)=1$ are more distinguishing, the power of $L R_{2 n}^{*}$ is less than that of $L R_{n}$ or $L R_{1 n}^{*}$ in all cases. This suggests that the bootstrapped critical values may be more precise than the critical values in Chan (I9.9I) for the $L R_{n}^{*}$ test. When the distance between $r_{L}$ and $r_{U}$ is small, $L R_{n}$ is less powerful than $L R_{1 n}^{*}$, and this power advantage grows as the distance between $r_{L}$ and $r_{U}$ becomes large. As we expected, this is because $L R_{n}$ (or $L R_{n}^{*}$ ) is the LR test 


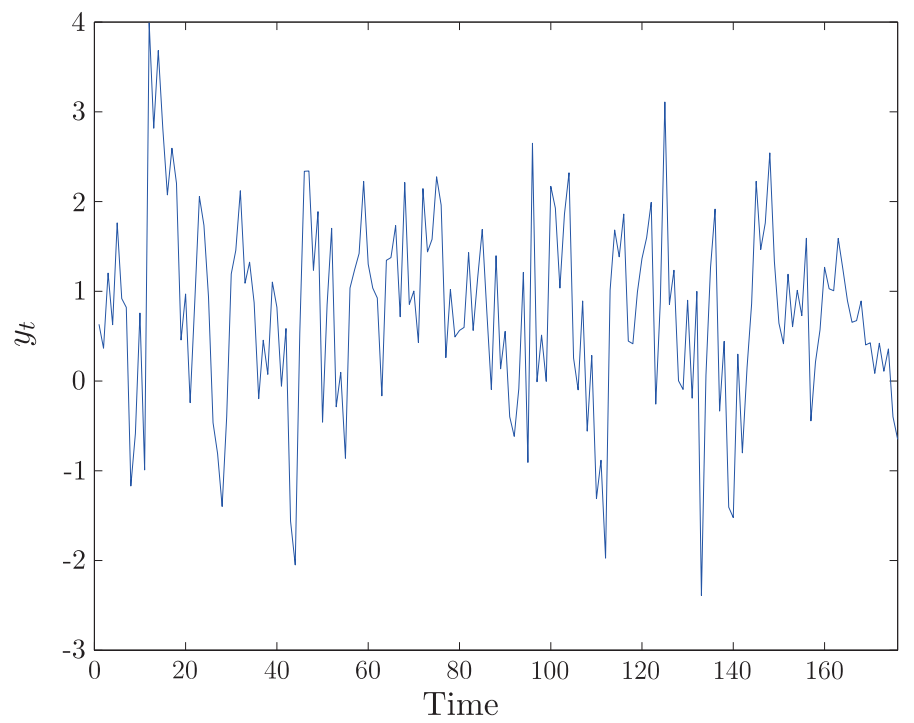

Figure 1. 100 times log-return of quarterly U.S. real GNP (in 1982 dollars) from the first quarter of 1947 to the first quarter of 1991.

when $r_{L}$ and $r_{U}$ are far from (or closed to) each other. The simulation results show that $L R_{n}$ performs well, especially when the buffering region is wide.

Next, we study the quarterly U.S. real GNP (in 1982 dollars) from the first quarter of 1947 to the first quarter of 1991. The 100 times log-return, denoted by $\left\{y_{t}\right\}$, has a total of 176 observations; see Figure 1 . We apply our test $L R_{n}$ and the LR test $L R_{n}^{*}$ in Chan ([1990) to this data set. The results with different values of $p$ and $d$ are reported in Table 2. From Table 2, we find that a marginal threshold effect can be detected at the $5 \%$ significance level in either the BAR or TAR model with $p=d=2$. Our finding is consistent with those in Potter (1995) and Hansen (1996), in which they also detected a marginal threshold effect in the TAR model by using the sup-LM test. Hence, we fit $\left\{y_{t}\right\}$ by the following two specifications:

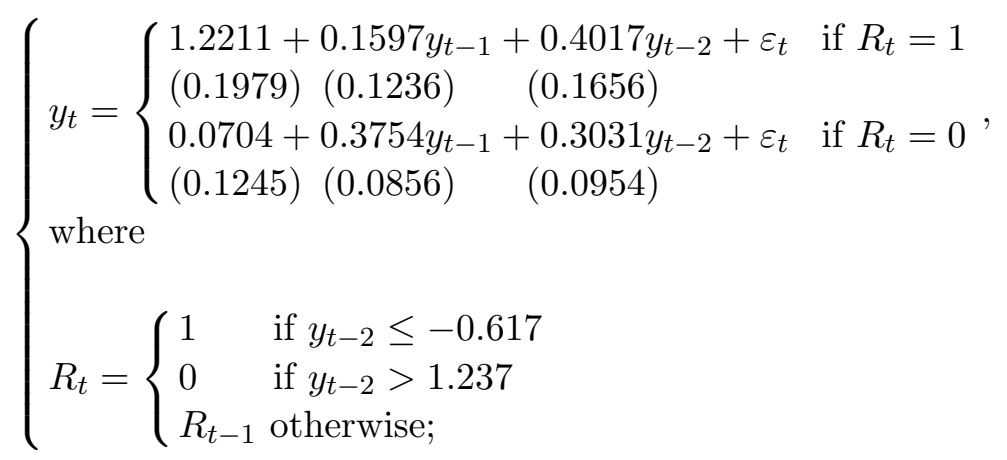


Table 2. Results of tests applied to data set $\left\{y_{t}\right\}^{\dagger}$.

\begin{tabular}{|c|c|c|c|c|c|c|c|c|c|}
\hline \multirow[b]{2}{*}{$p$} & \multirow[b]{2}{*}{$d$} & \multicolumn{4}{|c|}{ BAR model } & \multicolumn{4}{|c|}{ TAR model } \\
\hline & & $L R_{n}$ & $c_{0.1}$ & $c_{0.05}$ & $c_{0.01}^{\S}$ & $L R_{n}^{*}$ & $c_{0.1}^{*}$ & $c_{0.05}^{*}$ & $c_{0.01}^{*}{ }^{\S}$ \\
\hline 1 & 1 & 4.29 & 13.66 & 16.51 & 23.29 & 4.29 & 9.69 & 11.79 & 18.58 \\
\hline 2 & 1 & 9.08 & 17.97 & 22.07 & 30.76 & 5.83 & 14.57 & 17.75 & 24.92 \\
\hline 2 & 2 & $21.08^{\ddagger}$ & 18.53 & 21.36 & 29.58 & $13.69^{\ddagger}$ & 12.47 & 14.52 & 18.82 \\
\hline 3 & 1 & 7.18 & 20.88 & 23.93 & 31.63 & 6.46 & 15.60 & 19.10 & 26.02 \\
\hline 3 & 2 & 18.15 & 21.34 & 24.62 & 31.70 & 13.84 & 14.59 & 16.70 & 21.92 \\
\hline 3 & 3 & 14.38 & 20.07 & 23.67 & 32.50 & 8.16 & 17.02 & 20.83 & 30.15 \\
\hline
\end{tabular}

${ }^{\dagger}$ The value of $a$ and $b$ are set to be the 10th and 90th quantiles of $\left\{y_{t}\right\}$.

$\ddagger$ The p-values for $L R_{n}$ and $L R_{n}^{*}$ are 0.053 and 0.064 , respectively.

$\S c_{\alpha}\left(\right.$ or $c_{\alpha}^{*}$ ) is obtained by the bootstrap method in Section 3 with $J=1,000$.

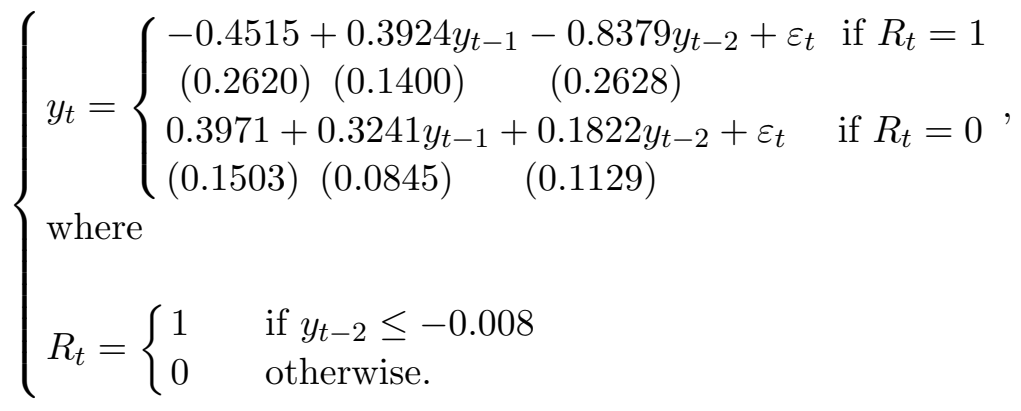

Models (4.2) and (4.3) are estimated by the least squares method (standard errors are in parentheses, and estimated values of $\sigma_{\varepsilon}^{2}$ are 0.85 and 0.90 , respectively). For model (4.2), the first 20 autocorrelations or partial autocorrelations of the residuals $\left\{\hat{\varepsilon}_{t}\right\}$ or $\left\{\hat{\varepsilon}_{t}^{2}\right\}$ are not significant at the $5 \%$ level; see Figure 2. Similar results hold for model (4.3), and they are not reported here. This suggests that both models are adequate to fit $\left\{y_{t}\right\}$. The values of log-likelihood for models $(4.2)$ and $(4.3)$ are -233.1 and -237.3 , respectively, and hence a $\operatorname{BAR}(2)$ model is more suitable than $\operatorname{TAR}(2)$ model to fit $\left\{y_{t}\right\}$.

Models (4.2) and (4.3) basically tell us different stories. Following Tiao and Tsay (19.94), if we treat a negative growth in GNP as 'contraction' and a positive growth as 'expansion', model (4.2) shows that the region of $y_{t}$ does not shift unless we have experienced a big 'contraction' or 'expansion' two years before, while model (4.3) indicates that the region of $y_{t}$ almost fully relies on the kind of economic status that we have at that time. Society or government may not have a large or quick response to a moderate growth in GNP, and hence the region of $y_{t}$ is most likely unchanged in this case. Thus, based on these facts, it is fair to conclude that a $\operatorname{BAR}(2)$ model is more reasonable than $\operatorname{TAR}(2)$ model to fit $\left\{y_{t}\right\}$. 
(a)

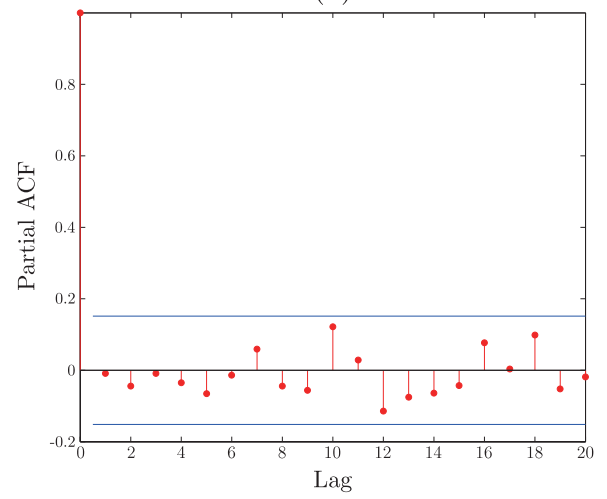

(c)

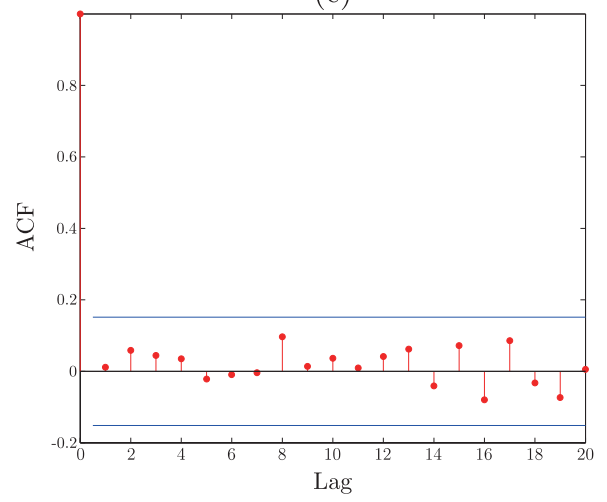

(b)

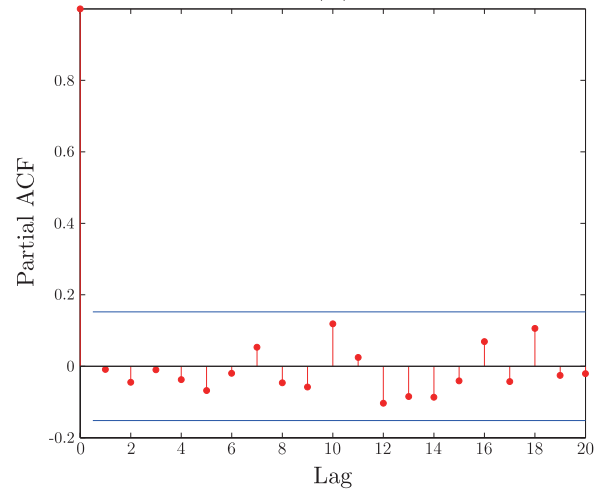

(d)

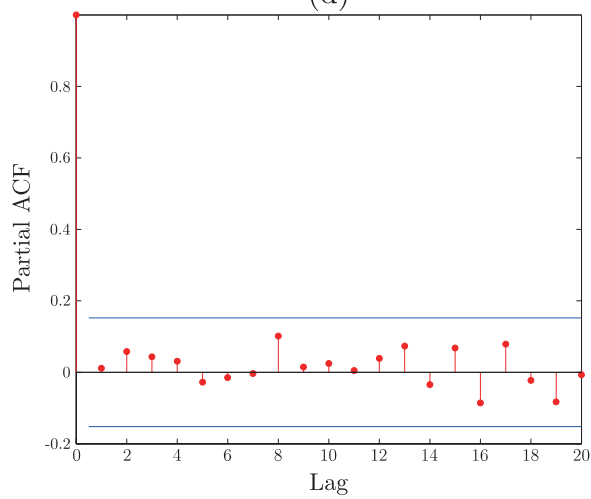

Figure 2. (a) the autocorrelations for $\left\{\hat{\varepsilon}_{t}\right\} ;(b)$ the partial autocorrelations for $\left\{\hat{\varepsilon}_{t}\right\} ;(c)$ the autocorrelations for $\left\{\hat{\varepsilon}_{t}^{2}\right\}$; and $(d)$ the partial autocorrelations for $\left\{\hat{\varepsilon}_{t}^{2}\right\}$.

In the end, it is also of interest to fit $\left\{y_{t}\right\}$ by a three-regime TAR model:

$$
y_{t}=\left\{\begin{array}{l}
-0.4969+0.3735 y_{t-1}-0.8500 y_{t-2}+\varepsilon_{t} \text { if } y_{t-2} \leq-0.288 \\
\begin{array}{l}
0.3649)(0.1399) \\
-3.3614+1.1691 y_{t-1}-15.872 y_{t-2}+\varepsilon_{t}
\end{array} \text { if }-0.288<y_{t-2} \leq-0.058 \\
(1.2807)(1.0193) \quad(4.3454) \\
0.3837+0.3233 y_{t-1}+0.1908 y_{t-2}+\varepsilon_{t} \quad \text { if } y_{t-2}>-0.058 \\
(0.1439)(0.0818) \quad(0.1083)
\end{array} .\right.
$$

Model (4.4) is estimated by the least squares method (standard errors in parentheses, and the estimated value of $\sigma_{\varepsilon}^{2}$ is 0.84 ). Model (4.4) may also be adequate to fit $\left\{y_{t}\right\}$ by looking at the first 20 autocorrelations and partial autocorrelations of the residuals $\left\{\hat{\varepsilon}_{t}\right\}$ and $\left\{\hat{\varepsilon}_{t}^{2}\right\}$. However, the number of effective observations for these regimes from lower to upper are 25, 10, and 139, respectively. Thus, 
although the value of log-likelihood for model (4.4) is -231.6, greater than that for model (4.2), a model with two regimes for $\left\{y_{t}\right\}$ seems more likely. We prefer to fit $\left\{y_{t}\right\}$ by a $\operatorname{BAR}(2)$ model in view of this point.

\section{Acknowledgements}

The authors greatly appreciate the helpful comments of Dr. G.D. Li, two anonymous referees, an Associate Editor, and the Editor Qiwei Yao. The authors would to thank the Research Grants Council of the Hong Kong SAR Government, GRF grant HKU703711P, for partial support. The first author's research is also supported by NSFC(No.11201459) and the National Center for Mathematics and Interdisciplinary Sciences, CAS.

\section{References}

Andrews, D. W. K. (1993a). Tests for parameter instability and structural change with unknow change point. Econometrica 61, 821-856.

Andrews, D. W. K. (1993b). An introduction to econometric applications of functional limit theory for dependent random variables. Econometric Rev. 12, 183-216.

Caner, M. and Hansen, B. E. (2001). Threshold autoregression with a unit root. Econometrica 69, 1555-1596.

Chan, K. S. (1990). Testing for threshold autoregression. Ann. Statist. 18, 1886-1894.

Chan, K. S. (1991). Percentage points of likelihood ratio tests for threshold autoregression. J. Roy. Statist. Soc. Ser. B 53, 691-696.

Chan, K. S. and Tong, H. (1990). On likelihood ratio tests for threshold autoregression. J. Roy. Statist. Soc. Ser. B 52, 469-476.

Davies, R. B. (1977). Hypothesis testing when a nuisance parameter is present only under the alternative. Biometrica 64, 247-254.

Davies, R. B. (1987). Hypothesis testing when a nuisance parameter is present only under the alternative. Biometrica 74, 33-43.

Doukhan, P., Massart, P. and Rio, E. (1995). Invariance principles for absolutely regular empirical processes. Annales de I'Institut H. Poincare 31 393-427.

Hansen, B. E. (1996). Inference when a nuisance parameter is not indentified under the null hypothesis. Econometrica 64, 413-430.

Hansen, B. E. (1999). Threshold effects in non-dynamic panels: Estimation, testing, and inference. J. Econom. 93, 345-368.

Li, G. D., Guan, B., Li, W. K. and Yu, P. L. H. (2012). Buffered threshold autoregressive time series models. Working paper. University of Hong Kong.

Li, G. D. and Li, W. K. (2008). Testing for threshold moving average with conditional heteroscedasticity. Statist. Sinica 18, 647-665.

Li, G. D. and Li, W. K. (2011). Testing a linear time series models against its threshold extension. Biometrika 98, 243-250.

Li, D. and Ling, S. (2013). On a threshold double autoregressive model. Working paper. Hong Kong University of Science and Technology. 
Ling, S. and Tong, H. (2005). Testing a linear MA model against threshold MA models. Ann. Statist. 33, 2529-2552.

Pham, T. D. and Tran, L. T. (1985). Some mixing properties of time series models. Stochastic Process. Appl. 19, 297-303.

Potter, S. M. (1995). A nonlinear approach to U.S. GNP. J. Appl. Econom. 10, 109-125.

Stute, W. (1997). Nonparametric model checks for regression. Ann. Statist. 25, 613-641.

Tiao, G. C. and Tsay, R. S. (1994). Some advances in non-linear and adaptive modelling in time-series. J. Forecasting 13, 109-131.

Tong, H. (1978). On a threshold model. In Pattern Recognition and Signal Processing (Edited by C. H. Chen), 101-141. Sijthoff and Noordhoff, Amsterdam.

Tong, H. (1990). Non-linear Time Series. A Dynamical System Approach. Clarendon Press, Oxford.

Tong, H. (2011). Threshold models in time series analysis-30 years on (with discussions). Statist. and Its Interface 4, 107-135.

Tsay, R. S. (1989). Testing and modeling threshold autoregressive processes. J. Amer. Statist. Assoc. 84, 231-240.

Tsay, R. S. (1998). Testing and modeling multivariate threshold models. J. Amer. Statist. Assoc. 93, 1188-1202.

Wong, C. S. and Li, W. K. (1997). Testing for threshold autoregression with conditional heteroscedasticity. Biometrika 84, 407-418.

Wong, C. S. and Li, W. K. (2000). Testing for double threshold autoregressive conditional heteroscedastic model. Statist. Sinica 10, 173-189.

Zhu, K. and Ling, S. (2012). Likelihood ratio tests for the structural change of an AR(p). model to a threshold AR(p). model. J. Time Ser. Anal. 33, 223-232.

Zhu, K., Yu, P. L. H. and Li, W. K. (2013). Testing for the buffered autoregressive processes (Supplementary Material).

Chinese Academy of Sciences, Institute of Applied Mathematics, HaiDian District, Zhongguancun, Bei Jing, China.

E-mail: kzhu@amss.ac.cn

Department of Statistics and Actuarial Science, University of Hong Kong, Pokfulam Road, Hong Kong.

E-mail: plhyu@hku.hk

Department of Statistics and Actuarial Science, University of Hong Kong, Pokfulam Road, Hong Kong.

E-mail: hrntlwk@hku.hk 